\title{
A Concurrent Newsvendor Problem with Rationing
}

\author{
Pinto Roberto \\ CELS - Research Center on Logistics and After-Sales Services \\ University of Bergamo \\ Viale Marconi, 5 - 24044 Dalmine, Italy \\ roberto.pinto@unibg.it
}

\begin{abstract}
The model proposed in this paper aims at identifying the best allocation of a limited quantity of product to a group of retailers supplied by a unique supplier, considering the variability of the demands faced on the market. The proposed approach aims at reducing the problem of demand inflation, inherent to many rationing policies, by considering the mean and the standard deviation of the demand faced by each retailer. Moreover it could provide managerial insights for managing concurrent retailers under availability constraints.
\end{abstract}

Keywords: Newsvendor problem, rationing, probabilistic demand, stock allocation, inventory management.

\section{Introduction}

The newsvendor (or newsboy, or single-period) problem (NVP hereafter) is a wellknown operations research model for controlling the inventory of a single item with stochastic demands over a single period i.e. when there is a single purchasing opportunity before the start of the selling period and the demand for the item is random.

It is assumed that a retailer places an order to a supplier for a finite quantity. In such a setting the size of the order should be carefully determined: in fact, if the order quantity is smaller than the realized demand, the newsvendor forgoes some profit. On the other hand, if any inventory remains at the end of the period, a discount is used to sell it or it is disposed of. Hence, the trade-off for the retailer is between the risk of overstocking (forcing disposal below the unit purchasing cost) and the risk of understocking (losing the opportunity of making a profit) [4]. The NVP aims at finding the order quantity that either maximizes the expected profit or minimizes the expected costs of overestimating and underestimating probabilistic demand when there is a single purchasing opportunity before the start of the selling period [9]. Indeed, both approaches yield the same results [8].

The importance of this problem is reflective of many real life situations and is often used to aid decision making in the fashion and sporting industries, both at the manufacturing and retail levels. Moreover, the NVP is not limited to inventory control: it can also be used in managing capacity and evaluating advanced booking of orders in service industries such as airlines and hotels [8]. 
Several extensions of the classical single-period / single product problem (dating back to early ' 50 with the contributes of Arrow et al. [1] and Morse and Kimbal [10]) have been proposed, in order to deal with multiperiod / multiproduct / multistage problems, different objectives and utility functions, different supplier pricing policies, different newsvendor pricing policies and discounting structures, different states of information about demand, constrained multi-products, multiple-products with substitution, random yields, and multi-location models [8].

However, the general NVP does not take into account the interaction of several newsvendors that can generate shortage at supplier level when a limited product quantity is available, with the consequent impossibility to completely fulfill the demand. For this reason, the present paper deals with a problem that can be considered as a further extension to the classical NVP: we consider several concurrent retailers (newsvendors) that face probabilistic demands expressed as probability density functions (for the sake of simplicity, in this paper we consider the Normal distribution). These retailers submit their orders for the same product to the same (unique) supplier.

In turn, the supplier has a limited quantity available for selling: when demand is uncertain and capacity is costly, a supplier will not build an amount of capacity sufficient to cover every possible demand realization. Hence problems arise when the overall amount of received orders from retailers exceeds the available quantity. In such a setting, the supplier must employ a rationing mechanism to allocate the available quantity among the retailers in order to maximize the expected profit (or minimize the expected cost) of the whole distribution system under probabilistic demand [3].

We implicitly assume that the objective of the supplier is to capture as much demand as possible at the customer level, considering that the selling price is the same for all the retailers. A typical example of the system described above is found when a franchise contracts between supplier and retailers are in place.

Several rationing policies have been proposed in literature. For example, proportional allocation based on percentage of total orders is perhaps the most intuitive scheme for dividing capacity, but it incentives the inflation of the order size: retailers order more than they desire in an attempt to ensure that their ultimate allocation is close to what they truly want [3].

The proposed model aims at overcoming this problem: in fact, the proposed model allocates the quantity considering the distribution of the demand faced by the retailers, instead of the actual size of the orders they could place.

In general rationing schemes, there is a rank of the incoming demand, such that these demand classes may have different values, so that it is more important to satisfy one class than the others; when the inventory is low, it is reasonable to ration inventory by rejecting demand from the less valuable classes in anticipation of future demand from the more valuable classes.

Even though this is quite a common setting, we focus our attention on the case where the value of each demand (retailer) is the same, and the only objective is to capture as much demand as possible at the customer level. Hence, in the described case we reject order from retailers that have less probability of selling the entire, requested quantity, the probability being based on the demand distribution expressed as known mean and standard deviation. 
The rationing decision naturally depends on the current inventory level as well as the operating characteristics of the system. For example, if the production leadtime is long and hence inventory cannot be replenished quickly, rationing will be more critical [5].

\subsection{The General Newsvendor Problem}

Formally, the general newsvendor problem is stated as follows. A decision maker (a retailer) places an order for a quantity $Q$, which arrives before the start of the selling period. Let $D$ be the stochastic demand during this period and let $\mu$ be its mean. Let $F$ be the distribution function of demand and $f$ the density function. For simplicity, assume $F$ is continuous, differentiable and strictly increasing. Hence, we can write:

$$
F(Q)=\operatorname{Prob}(0 \leq D \leq Q)=\int_{0}^{Q} f(x) d x
$$

Further, assume that the decision maker has an unbiased forecast of the demand distribution and knows $F$. The decision maker purchases each unit for cost $c$ and sells each unit at price $p>c$. When $Q>D$, each unit remaining at the end of the period can be salvaged for $s<c$ (notice that in the case the units are simply disposed, $s=0$ ). In case of shortage the company incurs a penalty cost per unit $v$.

If $Q$ units are ordered, then $\min (Q, D)$ units are sold and $\max (Q-D, 0)$ units are salvaged (disposed).

Hence, we define

$$
\pi(x)= \begin{cases}(p-c) \cdot Q-v \cdot(x-Q) & \text { if } x \geq Q \\ p \cdot x+s \cdot(Q-x)-c \cdot Q & \text { if } x<Q\end{cases}
$$

as the realized profit, and let

$$
\begin{gathered}
E[\pi(x)]=(p+s-c) \int_{Q}^{\infty} Q f(x) d x-v \int_{Q}^{\infty} x f(x) d x+ \\
+(p-s) \int_{0}^{Q} x f(x) d x-(c-s) \int_{0}^{Q} Q f(x) d x
\end{gathered}
$$

be the expected profit. Using Leibniz's rule to obtain the first and second derivatives it is possible to show that $E[\pi]$ is concave; hence the sufficient optimality condition is the well known fractile formula [8], [11]:

$$
F\left(Q^{*}\right)=\frac{p+v-c}{p+v-s} \quad \rightarrow \quad Q^{*}=F^{-1}\left(\frac{p+v-c}{p+v-s}\right)
$$

\section{Design of the Proposed Approach}

The problem we are considering is a single period / 1 -warehouse / $N$-retailer inventory system where demand occurs at all locations with the same probability distribution (but with different mean and standard deviation). We assume that demands coming from different retailers are equally important; hence, the inventory manager have to apply some type of rationing policy based on the probability of reaching as much demand as possible at customers level, when the total quantity required exceeds the available quantity at the supplier level. 
For the sake of clarity, we distinguish two stages. The first stage is called ordering stage: each retailer places its own order (solving its own NVP) of size $Q_{i}$. Hence, from the supplier's standpoint this is comparable to $N$ instances of independent NVPs (being $N$ the number of ordering retailers). The solutions of these independent problems represent the best order size for each retailer and a bound for the overall solution.

Since each retailer makes his decision independently, there is no guarantee that the sum of the order sizes does not exceed the supplier's availability $A$. Therefore, if $\sum_{i} Q_{i}>A$ a rationing procedure takes place in the second stage.

In the second stage, called rationing stage, a set of constraints is imposed in order to bind the independent solutions to the available quantity $A$. From a mathematical standpoint we can state the problem as:

$$
\begin{gathered}
\min E[c, s, v, \bar{Q}] \text { s.t. } \\
\sum_{i} Q_{i} \leq A \text { and } Q_{i} \geq 0 \quad \forall i=\left\{Q_{i} \in \bar{Q}\right\}
\end{gathered}
$$

The mathematical formulation appears really simple, indeed, but since it generally implies a non-linear distribution function in the expected value operator, the problem is non-linear. Hence, an alternative approach has been devised. In the remainder we present the procedure for the rationing stage.

\subsection{Rationing Stage}

In the rationing stage, the order sizes proposed by retailers are modified in order to take into account the availability at the supplier level. In doing this we want to assure to cover as much demand as possible at the customer level, allocating the right portion of $A$ to each retailer.

We start expressing the demand as:

$$
Q_{i}=\mu_{i}+k_{i} \cdot \sigma_{i}
$$

where $\mu_{i}$ and $\sigma_{i}$ are the mean and the standard deviation of the demand faced by the $i$ th retailer and $k_{i}$ a multiplicative factor, not yet know at this point. We use this representation according to the Chebyshev inequality:

$$
\operatorname{Prob}(|D-\mu|<k \cdot \sigma) \geq 1-\frac{1}{k^{2}}
$$

In other words, given a set of values $D=\left(d_{1}, \ldots, d_{n}\right)$ and a number $k$, at least a percentage of $1-1 / k^{2}$ falls in the interval $(\mu \pm k \cdot \sigma)$. Hence, maneuvering $k$ according to (7) we can assure that a given proportion of the demand will be covered.

In order to find $k_{i}$ we have to solve the following equation:

$$
\sum_{i} Q_{i}=A \rightarrow \sum_{i} k_{i} \cdot \sigma_{i}=A-\sum_{i} \mu_{i}
$$

Hence we have $N$ incognitos $\left(k_{i}\right)$ and only 1 equation. No others intuitive equations seem to be available in order to close the equation system, therefore other numerical approaches have been tested. 
A first attempt was performed using a Monte Carlo simulation, by randomly generating the value of $k_{i}$ in a given range (i.e. [0; 4] in order to cover at least $93 \%$ of demand, according to (7)). But in this way we cannot assure that the constraint expressed in (8) holds.

Hence, in order to solve this system we have to close it by letting an incognita depending by the other values. We can rewrite (8) as:

$$
k_{j}=\frac{A-\sum_{i} \mu_{i}-\sum_{i \neq j} k_{i} \cdot \sigma_{i}}{\sigma_{j}}
$$

where $j$ is the index of one of the $N$ retailers.

At this point, the mean $\mu_{i}$ are known and we can try different values of $k_{i}$ (for $\mathrm{i} \neq \mathrm{j}$ ) being sure that the overall constraint (8) will be always satisfied. In other words, $k_{j}$ is assumed as a balancing variable that assure the satisfaction of the constraint.

In the Monte Carlo simulation we can draw $N-1$ values for $k_{i}(i \neq j)$ and then calculate $k_{j}$ in order to satisfy the constraint. Clearly we do not accept the solutions in which $Q_{i}<0$; in fact, only the sum of $Q_{i}$ is checked against the availability $A$, while no check is performed in order to assure that $Q_{i}>0$.

While Monte Carlo simulation allows the exploration of the search space, it does not assure a monotone descent towards the minimum (since the solution are drawn randomly, there is no a monotone sequence of solutions towards the optimum).

Hence we decided to devise and test a Genetic Algorithm (GA) approach. In the GA, a population of solutions evolve from one iteration to the following improving quasi-monotonically (that is, there could be cases where the best solution in the $m+1$ iteration is the same of the best solution in the $m$-th iteration).

\section{Improving the Solution Procedure Using GA}

Genetic algorithm (GA) is a well known method for solving optimization problems that mimic natural selection, repeatedly modifying a population of individuals that represent solutions to the problem at hand. Each individual encodes a solution. At each step, the genetic algorithm selects individuals from the current population and uses them produce the children for the next generation. Over successive generations, the population evolves towards an optimal solution. It is recognized that in some classes of problem it makes possible to explore a far greater range of potential solutions to a problem than do conventional programs.

The GA uses three main types of rules at each step to create the next generation from the current population: (i) selection rules to select the individuals, called parents, that contribute to the population at the next generation; (ii) crossover rules, used to combine two parents to form children for the next generation; (iii) mutation rules that apply random changes to individual parents to form children, allowing to expand the search space introducing stochastic variability.

We implemented the GA using the Matlab GA Toolbox ${ }^{\mathrm{TM}}$. Several options are available for the most important parameter: we briefly report only the best choices for the GA parameters as resulted from an extensive test run: 
- Encoding: the population is encoded as a vector of $N-1$ double values, representing the maneuvered $k_{i}$. This seems the most reasonable choice in this case, but at the same time it implies other constraints in the choice of other operators such as mutation and crossover [6].

- Selection: the Stochastic uniform function lays out a line in which each parent corresponds to a section of the line of length proportional to its value. The algorithm moves along the line in steps of equal size. At each step, the algorithm allocates a parent from the section it lands on.

- Mutation: the Gaussian mutation function adds a random number taken from a Gaussian distribution with mean 0 to each entry of the parent vector.

- Crossover: the crossover function creates children by taking a weighted average of the selected parents.

For the sake of clarity, although these choices stem from a test run, at present we only exploited Matlab ${ }^{\mathrm{TM}}$ built-in functions: it could be possible to obtain better results implementing custom operators, but this aspect goes beyond the scope and purpose of this paper.

\section{Computational Results}

The proposed approach has been tested on different problem settings, from small (10 retailers) to medium (50 retailers) to large (100 retailers). We just report some main results on three specific settings that differ in the values of the mean and standard deviation. Each setting is run with increasing values of $A$ in the interval $\left[\sum \mu-\sum \sigma\right.$; $\left.\sum \mu+\sum \sigma\right]$. For each value of $A$ the GA determines the optimal allocation between $N$ retailers. For the sake of simplicity, we present the results for $N=3$.

\section{Setting 1: retailers with same mean and same standard deviation}

When all retailers face the same type of demand (i.e. they face the same demand distribution with the same mean and standard deviation) the best solution appears to be the one that maximize imbalance between retailers when availability is below a threshold; after such threshold, the best solution is obtained by minimizing the imbalance. From the test executed, the threshold can be empirically set at about $N \cdot \mu$ $+\sigma$, but this value deserves a deeper analysis.

\section{Setting 2: retailers with same mean and different standard deviation}

When all the retailers face the same demand distribution with the same mean but with different standard deviations, the best solution is to give higher priority to retailers that face demand with lower standard deviations. As in the previous case, it has been empirically observed that as the availability $A$ increases, the imbalance in the allocation tends to decrease.

\section{Setting 3: retailers with different mean and different standard deviation}

In this case we considered two sub cases: in the first one the standard deviation is proportional to the mean, while in the second case the standard deviation is inversely proportional to the mean. 
In the first sub case, as the availability increases, the proportion allocated to the retailers with higher standard deviation increases, while the proportions allocated to other retailers remains almost constant.

In the second sub case, the retailer with highest mean demand and lowest standard deviation is favored, while retailer with lowest demand and highest standard deviation is backlogged. As the availability increases, the quantity given to the latter rapidly increases, while the quota devoted to the others is kept almost constant.

\section{Convergence issue on large problems}

The approach has been tested on large instances (with 50 and 100 retailers). As expected, the GA converges to a solution slower as the number of retailer increases. Moreover, depending on the shape of the fitness landscape, the convergence to global optima is generally not assured.

\section{Conclusion, Research Limitations and Extensions}

In its present form, the paper presents a formulation and a solution procedure based on Genetic Algorithm to a problem where several newsvendors compete for the same resource provided by a unique supplier.

GA may have a tendency to converge towards local optima or even arbitrary points rather than the global optimum of the problem; hence further solution heuristics are currently under development and testing.

The presented problem does not consider cooperation or information sharing among retailers (which is considered in other, recent works, such that of Huang and Iravani [7]): retailers operate as isolated entities and should decide on their own the size of the order before the beginning of the selling season (ordering stage).

The proposed formulation of the problem is limited by the fact that it only considers the cost of inventory neglecting the service level at the retailer and customer level. Further extensions of the proposed model will encompass the possibility to set different service levels for each retailer (as in [2]). Another extension would encompass the possibility to handle several demand classes with different values so that it is more important to satisfy one class than the others.

\section{References}

1. Arrow, K.A., Harris, T.E., Marschak, J.: Optimal inventory policy. Econometrica 19, 250 $272(1951)$

2. Axsäter, S., Kleijn, M., De Kok, T.G.: Stock Rationing in a Continuous Review TwoEchelon Inventory Model. Annals of Operations Research 126, 177-194 (2004)

3. Cachon, G.P., Lariviere, M.A.: An equilibrium analysis of linear, proportional and uniform allocation of scarce capacity. IIE Transactions 31, 835-849 (1998)

4. Gallego, G., Moon, I.: The distribution free newsboy problem: review and extensions. Journal of operational research society 44(8), 825-834 (1993)

5. Ha, A.Y.: Inventory Rationing in a Make-to-Stock Production System with Several Demand Classes and Lost Sales. Management Science 43, 1093-1103 (1997) 
6. Herrera, F., Lozano, M., Verdegay, J.L.: Tackling Real-Coded Genetic Algorithms: Operators and Tools for Behavioural Analysis. Artificial Intelligence Review 12, 265-319 (1998)

7. Huang, B., Iravani, S.M.R.: Optimal production and rationing decisions in supply chains with information sharing. Operations Research Letters 35, 669-676 (2006)

8. Khouja, M.: The single-period (news-vendor) problem: literature review and suggestions for future research. Omega International Journal of Management Science 27, 537-553 (1999)

9. Kogan, K., Lou, S.: Multi-stage newsboy problem: A dynamic model. European Journal of Operational Research 149, 448-458 (2003)

10. Morse, M.P., Kimbal, G.E.: Methods of Operations Research. MIT Press, Cambridge (1951)

11. Schweitzer, M.E., Cachon, G.P.: Decision Bias in the Newsvendor Problem with a Known Demand Distribution: Experimental Evidence. Management Science 46(3), 404-420 (2000) 